\title{
Telecommunication Address Type
}

National Cancer Institute

\section{Source}

National Cancer Institute. Telecommunication Address Type. NCI Thesaurus. Code C70955.

A particular category of telecommunication address regarded as having particular characteristics, qualities, type of usage, and defined similarities. 